\title{
Proteins associated with EGFR-TKIs resistance in patients with non-small cell lung cancer revealed by mass spectrometry
}

\author{
SHAOYU YANG ${ }^{1 *}$, XUEQIN CHEN $^{1 *}$, YUELONG PAN $^{1}$, JIEKAI YU $^{2}, \mathrm{XIN} \mathrm{LI}^{1}$ and SHENGLIN MA ${ }^{1}$ \\ ${ }^{1}$ Department of Medical Oncology, Nanjing Medical University, Affiliated Hangzhou Hospital, \\ Hangzhou First People's Hospital, Hangzhou, Zhejiang 310006; ${ }^{2}$ Cancer Institute, Second Affiliated Hospital, \\ School of Medicine, Zhejiang University, Hangzhou, Zhejiang 310009, P.R. China
}

Received September 6, 2015; Accepted September 13, 2016

DOI: $10.3892 / \mathrm{mmr} .2016 .5823$

\begin{abstract}
The present study aimed to identify potential serum biomarkers for predicting the clinical outcomes of patients with advanced non-small cell lung cancer (NSCLC) treated with epidermal growth factor receptor tyrosine kinase inhibitors (EGFR-TKIs). A total of 61 samples were collected and analyzed using the integrated approach of magnetic bead-based weak cation exchange chromatography and matrix-assisted laser desorption/ionization-time of flight-mass spectrometry. The Zhejiang University Protein Chip Data Analysis system was used to identify the protein spectra of patients that are resistant and sensitive to EGFR-TKIs. Furthermore, a support vector machine was used to construct a predictive model with high accuracy. The model was trained using 46 samples and tested with the remaining 15 samples. In addition, the ExPASy Bioinformatics Resource Portal was used to search potential candidate proteins for peaks in the predictive model. Seven mass/charge $(\mathrm{m} / \mathrm{z})$ peaks at 3,264, 9,156, 9,172, 3,964, 9,451, 4,295 and 3,983 Da, were identified as significantly different peaks between the EGFR-TKIs sensitive and resistant groups. A predictive model was generated with three protein peaks at 3,264, 9,451 and $4,295 \mathrm{Da}(\mathrm{m} / \mathrm{z})$. This three-peak model was capable of distinguishing EGFR-TKIs resistant patients from sensitive patients with a specificity of $80 \%$ and a sensitivity of $80.77 \%$. Furthermore, in a blind test, this model exhibited a high specificity $(80 \%)$ and a high sensitivity $(90 \%)$. Apelin, TYRO
\end{abstract}

Correspondence to: Dr Shenglin Ma, Department of Medical Oncology, Nanjing Medical University, Affiliated Hangzhou Hospital, Hangzhou First People's Hospital, 261 Huansha Road, Hangzhou, Zhejiang 310006, P.R. China

E-mail: masnil@163.com

*Contributed equally

Key words: non-small cell lung cancer, epidermal growth factor receptor tyrosine kinase inhibitors, matrix-assisted laser desorption/ionization-time of flight-mass spectrometry, predictive model protein tyrosine kinase-binding protein and big endothelin-1 may be potential candidates for the proteins identified with an $\mathrm{m} / \mathrm{z}$ of 3,264, 9,451 and 4,295 Da, respectively. The predictive model used in the present study may provide an improved understanding of the pathogenesis of NSCLC, and may provide insights for the development of TKI treatment plans tailored to specific patients.

\section{Introduction}

Lung cancer has been identified as one of the leading causes of cancer-associated mortality worldwide, and $80-85 \%$ of lung cancer cases are classified as non-small cell lung cancer (NSCLC) (1). Considerable progress has been made in the development of epidermal growth factor receptor tyrosine kinase inhibitors (EGFR-TKIs), such as erlotinib and gefitinib, which regulate the expression levels of specific molecules in lung cancer cells (2). Gefitinib monotherapy has become more popular as a treatment option for chemotherapy-refractory patients and is also considered a first-line treatment option in specific advanced cancers, including NSCLC $(3,4)$. However, after 6-12 months of treatment, a number of patients that initially responded to EGFR-TKIs treatment ultimately became resistant and underwent tumor progression. This phenomenon has been defined as 'acquired resistance', and is common in patients with NSCLC $(5,6)$. Therefore, acquired resistance to EGFR-TKIs is an important issue to overcome for the successful treatment of patients with NSCLC (7). A previous study was performed with the aim of investigating the underlying mechanisms and regulating acquired resistance; however, in $30 \%$ of cases it remained inevitable (8). Therefore, more arduous efforts should be made to identify effective diagnostic markers and to provide tailor-made TKI treatment for each patient with EGFR-TKIs resistance.

Previous studies have attempted to identify clinical and laboratory hematological biomarkers in order to distinguish patients with NSCLC that were resistant to EGFR-TKIs treatment (9-11). An EGFR mutation has been reported to be associated with increased EGFR-TKI sensitivity in patients with NSCLC, and $\sim 80 \%$ of patients exhibit a good response to EGFR-TKIs treatment when EGFR was mutated (12). However, performing EGFR mutational profiling at every treatment stage on patients with NSCLC 
is complicated, expensive and time-consuming (13). Blood testing is an alternative option for monitoring the treatment progress at a low cost and convenience; however, the sensitivity of this method is low (14). Matrix-assisted laser desorption/ionization-time of flight-mass spectrometry (MALDI-TOF MS) has been developed for the analysis of various biological specimens, including serum, urine and tissue samples $(15,16)$. MALDI-TOF MS is able to simultaneously investigate large quantities of proteins and identify the proteomic patterns with high sensitivity, and has been used to determine the proteomic patterns in NSCLC (17). Furthermore, the magnetic bead (MB)-based platform for proteomic profiling with high sensitivity has been used to screen for biomarkers of several types of cancer, including breast cancer, esophageal carcinoma and lung cancer, in combination with MALDI-TOF MS (18-20).

The present study aimed to investigate the serum biomarkers that may predict the efficacy of EGFR-TKIs treatment by combining MALDI-TOF MS and MB methods. In addition, a model to predict EGFR-TKIs targeted therapy resistance among patients with advanced NSCLC was established, and its accuracy and sensitivity were assessed using a blind test set.

\section{Materials and methods}

Patients and materials. A total of 61 patients with NSCLC treated at Hangzhou First People's Hospital (Hangzhou, China) between June 2009 and May 2014 were enrolled in the present study. The inclusion criteria were as follows: i) Patients with advanced NSCLC, identified as stage IV according to TNM staging (21); ii) patients with a performance status of 0-2, which was defined according to the Eastern Cooperative Oncology Group (22); and iii) patients accepted target therapy by one of three EGFR-TKIs, specifically erlotinib, gefitinib or icotinib. Written informed consent was obtained from all enrolled patients. Following treatment with EGFR-TKIs, patients with progressive disease or a stable disease $>6$ months were considered to be EGFR-TKIs resistant, whereas patients with a partial response or stable disease $<6$ months were considered to be EGFR-TKIs sensitive. The protocol of the present study was approved by the Institutional Ethics Committee of Hangzhou First People's Hospital.

Protein extraction and MALDI-TOF MS protocol. Blood samples $(2 \mathrm{ml})$ were collected at the Hangzhou First People's Hospital by venipuncture from the patients with advanced NSCLC prior to EGFR-TKIs treatment and stored at $4^{\circ} \mathrm{C}$ within $1 \mathrm{~h}$. Subsequently, the samples were centrifuged at $5,500 \times \mathrm{g}$ for $5 \mathrm{~min}$ at $4^{\circ} \mathrm{C}$. Serum samples were isolated from the whole blood, divided into $100 \mu \mathrm{l}$ aliquots and stored at $-80^{\circ} \mathrm{C}$ for future use.

MB-based weak cation exchange chromatography (MB-WCX; CM10 spin columns; Ciphergen Biosystems Inc., Freemont, CA, USA) was used for peptidome separation of samples according to the standard protocol of the manufacturer. Initially, $10 \mu \mathrm{l}$ magnetic suspension with weak cation was thoroughly mixed with $10 \mu \mathrm{l}$ binding solution in a $0.5 \mathrm{ml}$ microfuge tube. Subsequently, $5 \mu 1$ serum was added to each tube at room temperature. All the tubes were placed in an MB separator (MBS; Miltenyi Biotec, Inc., Cambridge, MA, USA) for $1 \mathrm{~min}$ and the MB was then collected from the wall of the tubes. Each sample was washed twice, and $5 \mu$ l eluting solution was added for each sample. MB was separated again from the suspension using the MBS and the supernatant was removed to another sample and was blended with $5 \mu$ l stabilization solutions. Finally, prior to the MALDI-TOF MS analysis, the targets were prepared by spotting $1 \mu 1$ proteome fraction obtained from MB-WCX on the polished steel target (Bruker Daltonik GmbH, Bremen, Germany). Following air drying, $1 \mu 1$ mixture solution, containing $3 \mathrm{mg} / \mathrm{ml} \alpha$-cyano-4-hydroxycinnamic acid in 50\% ACN and $50 \%$ Milli-Q (EMD Millipore, Billerica, MA, USA) with 2\% trifluoroacetic acid, was applied onto each spot and the target was air dried again in order to achieve co-crystallization. The peptide calibration standard ( $1 \mathrm{pmol} / \mu 1$ peptide mixture) was applied in order to calibrate the machine.

MS analysis. For proteome analysis, a linear Ultraflex MALDI-TOF MS (Bruker Daltonik $\mathrm{GmbH}$ ) was used at the following settings: Ion source $1,20.00 \mathrm{kV}$; ion source 2, $18.60 \mathrm{kV}$; lens, $6.60 \mathrm{kV}$; pulsed ion extraction, $120 \mathrm{~ns}$. Ionization was achieved by irradiation with a crystal laser operating at $200.0 \mathrm{~Hz}$. For matrix suppression, a high gating factor with signal suppression up to $600 \mathrm{Da}$ was used. Mass spectra were detected using linear positive mode. Mass calibration was conducted using the calibration mixture (TuneMix mixture; Bruker Daltonics $\mathrm{GmbH}$, Leipig, Germany) of proteins and peptides in the mass range of 1,000-20,000 Da. Three MALDI preparations (MALDI spots) were quantified for each MB fraction. Concurrently, a total of 1,600 spectra were acquired (200 laser shots at eight different spot positions) for each MALDI spot. Spectra were collected automatically through the Autoflex Analysis software (version 2.2; Bruker Daltonik $\mathrm{GmbH}$ ) to generate the optimized raw data by controlled adjustment of critical instrument settings.

The criteria selected for protein mass peak detection (mass/charge; $\mathrm{m} / \mathrm{z}$ ) were as follows: Signal-to-noise ratio $>3$ and a 2-Da peak width filter. The intensities of the interest peaks were normalized to total peak intensity. More than $10 \%$ of the molecular weight was sieved in simultaneous samples, with the discrepancy of identical peaks in different samples $<0.3 \%$ following the removal of initial data noise.

Protein identification and bioinformatics analysis. Zhejiang University Protein Chip Data Analysis system was used to identify the mass peaks that were significantly different between the sensitive and resistant patient groups. All of the peaks were analyzed by one-way analysis of variance, followed by a Student-Newman-Keuls post-hoc test using SPSS version 19.0 (IBM SPSS, Armonk, NY, USA). P $<0.05$ was considered to indicate a statistically significant difference. A predictive model using the marker proteins was constructed using a support vector machine (SVM), which was then trained and tested for prediction accuracy. Subsequently, ExPASy Bioinformatics Resource Portal (www.expasy.org/proteomics) (23) was used to detect potential candidate proteins for the protein peaks observed in the present study. 


\section{Results}

Clinical characteristics of the participants. Among the 61 patients enrolled in the present study, none had a complete response to EGFR-TKIs therapy: 18 patients had a partial response (29.5\%); 18 patients had stable disease for $<6$ months (29.5\%), whereas another 25 patients had progressive disease or stable disease for $>6$ months $(41 \%)$. There were 36 patients identified as sensitive to EGFR-TKIs and 25 patients identified as resistant to EGFR-TKIs. The clinical characteristics of the participants are presented in Table I. The median overall survival time in the sensitive group was significantly greater compared with the resistant group (19.56 \pm 1.97 vs. 8.41 \pm 1.97 months; $\mathrm{P}<0.05$; Fig. 1).

Comparison of mass spectra between EGFR-TKIs sensitive and resistant groups. A total of seven mass peaks were identified as significantly different between the sensitive and resistant groups: 3,264, 9,156, 9,172, 3,964, 9,451, 4,295 and 3,983 Da (Table II). Among them, the peaks at 9,156, 9,172, 3,964, 9,451, 4,295 and 3,983 Da were low in the resistant group and high in the sensitive group. However, the m/z peak at 3,264 Da was the only peak that was higher in the EGFR-TKIs resistant group compared with the EGFR-TKIs sensitive group.

Construction and validation of the predictive model. From the seven mass peaks, three were used for the construction of the SVM model with the highest accuracy. This model was used as a predictive model and consisted of three potential biomarkers with a $\mathrm{m} / \mathrm{z}$ ratio of $3,264,9,451$ and 4,295 $\mathrm{Da}$ (Fig. 2). The predictive model was trained with 46 samples and tested using the remaining 15 samples. Following the 10-fold cross-validation SVM, the three-peak model established in the training set was able to distinguish EGFR-TKIs sensitive patients from EGFR-TKIs resistant patients with a specificity of $80 \%$ and a sensitivity of $80.77 \%$. In the blind test sets, 9 out of 10 EGFR-TKIs sensitive samples and 4 out 5 EGFR-TKIs resistant samples were identified correctly with a specificity of $80 \%$ and a sensitivity of $90 \%$ (Table III).

The ExPASy Bioinformatics Resource Portal was used to identify candidate proteins for the three peaks. Apelin was identified as a potential candidate for the protein peak with an $\mathrm{m} / \mathrm{z}$ of 3,264 Da, which was the only high peak in the resistant group. TYRO protein tyrosine kinase-binding protein (TYROBP; 9,450 Da), and big endothelin-1 (big ET-1; $2,487 \mathrm{Da}$ ) may be the potential candidates for the proteins with an $\mathrm{m} / \mathrm{z}$ of 9,451 and $4,295 \mathrm{Da}$, respectively.

\section{Discussion}

For the successful clinical development of individual therapeutic strategies for patients with NSCLC, it is critical to identify biomarkers that may predict drug resistance in patients. MALDI-TOF MS, which is a high-throughput proteomic technique, has been widely used to increase the success of screening for novel biomarkers of numerous diseases $(24,25)$. In the present study, the protein fingerprints in blood serum samples from 36 EGFR-TKIs sensitive patients and 25 EGFR-TKIs resistant patients were analyzed. The combination of several biomarkers is considered to be
Table I. Clinical characteristics of the patients with non-small cell lung cancer included in the present study.

\begin{tabular}{lcc}
\hline Characteristic & Patients (n=61) & Percentage (\%) \\
\hline Gender & 35 & 57 \\
Male & 26 & 43 \\
Female & & \\
Age, years & 62 & $32-85$ \\
Median (range) & 31 & 51 \\
$\leq 62$ & 30 & 49 \\
>62 & & \\
Smoking history & 41 & 67 \\
Never & 20 & 33 \\
Current or past smoker & & \\
Histology & 55 & 10 \\
Adenocarcinoma & 6 & 43 \\
Others & & 90 \\
Patients treated with TKIs & 26 & 48 \\
Gefitinib & 6 & \\
Erlotinib & & \\
Icotinib & & \\
\hline
\end{tabular}

TKIs, tyrosine kinase inhibitors.

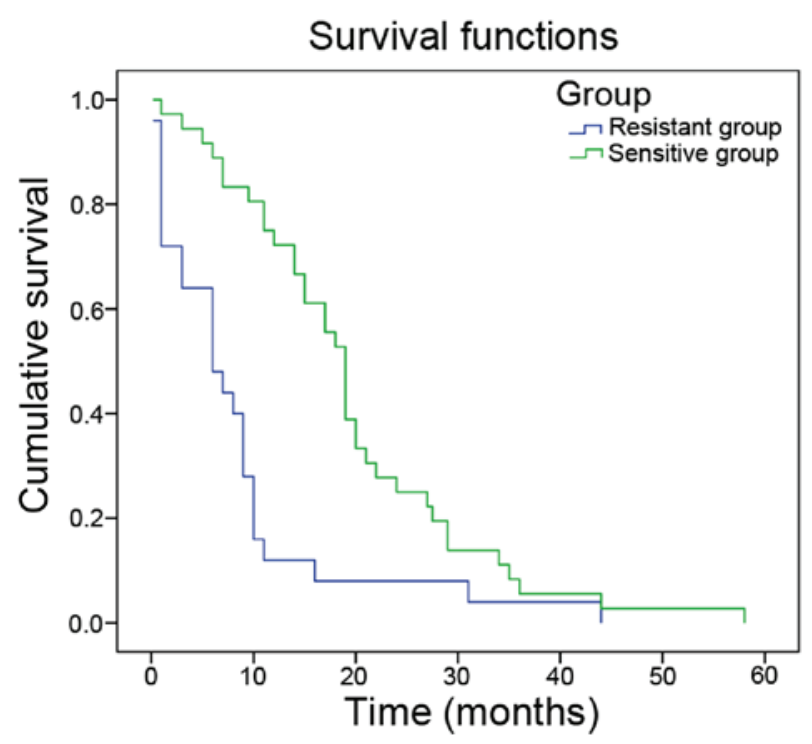

Figure 1. Overall survival for patients treated with epidermal growth factor receptor tyrosine kinase inhibitors in sensitive and resistant groups.

more reliable and powerful for diagnosis compared with the use of a single marker (18). The present study used the SVM method to construct a three protein peak model and screened samples to distinguish between EGFR-TKIs resistant and sensitive patients. Further analysis revealed apelin, TYROBP and big ET-1 may be the potential proteins in the predictive model.

Apelin is a 36 -amino acid peptide, which has a wide endogenous expression in various tissues, including the gastrointestinal tract, lung, heart, liver and bone $(26,27)$. Apelin has 
Table II. Distinct protein peaks between EGFR-TKIs sensitive group and resistant group.

\begin{tabular}{lccc}
\hline & \multicolumn{3}{c}{ Relative intensity } \\
\cline { 2 - 4 } $\begin{array}{l}\text { Intensity of } \\
\text { protein peak }(\mathrm{m} / \mathrm{z})\end{array}$ & $\begin{array}{c}\text { Sensitive group } \\
(\mathrm{n}=36, \text { mean } \pm \mathrm{SD})\end{array}$ & $\begin{array}{c}\text { Resistant group } \\
(\mathrm{n}=25, \text { mean } \pm \mathrm{SD})\end{array}$ \\
\hline 3,264 & $273.53 \pm 83.13$ & $425.52 \pm 200.28$ & 0.0025 \\
9,156 & $298.50 \pm 421.61$ & $141.07 \pm 130.07$ & 0.0162 \\
9,172 & $252.60 \pm 264.53$ & $139.56 \pm 123.41$ & 0.0206 \\
3,964 & $1,043.65 \pm 839.88$ & $516.94 \pm 470.90$ & 0.0275 \\
9,451 & $318.10 \pm 371.29$ & $190.33 \pm 121.18$ & 0.0426 \\
4,295 & $390.54 \pm 417.55$ & $213.61 \pm 360.41$ & 0.0449 \\
3,983 & $353.76 \pm 437.12$ & $242.72 \pm 472.91$ & 0.0473
\end{tabular}

EGFR-TKIs, epidermal growth factor receptor tyrosine kinase inhibitors; SD, standard deviation.

Table III. Predicted results of the model for discriminating targeted therapy resistance from targeted therapy sensitivity.

\begin{tabular}{|c|c|c|c|c|c|c|}
\hline \multirow[b]{2}{*}{ Group } & \multicolumn{3}{|c|}{$\begin{array}{l}\text { Training set } \\
\qquad(\mathrm{n}=46)\end{array}$} & \multicolumn{3}{|c|}{$\begin{array}{l}\text { Testing set } \\
\quad(n=15)\end{array}$} \\
\hline & S & $\mathrm{R}$ & Sum & S & $\mathrm{R}$ & Sum \\
\hline S & 16 & 4 & 20 & 4 & 1 & 5 \\
\hline $\mathrm{R}$ & 5 & 21 & 26 & 1 & 9 & 10 \\
\hline Sensitivity, \% & \multicolumn{3}{|c|}{80.77} & \multicolumn{3}{|c|}{90} \\
\hline Specificity, \% & \multicolumn{3}{|c|}{80} & \multicolumn{3}{|c|}{80} \\
\hline
\end{tabular}

$\mathrm{S}$, sensitive; R, resistant.

been shown to regulate glucose homeostasis and is closely associated with obesity (28). A previous study reported that apelin may act as a potential proangiogenic factor in various cancers (29). Increased apelin levels stimulate the microvessel densities and perimeters by suppressing the proliferation of endothelial cells in NSCLC $(30,31)$. Furthermore, apelin has been reported to be overexpressed in NSCLC, and its expression may influence the clinical outcomes of NSCLC (30). The present study demonstrated that the expression of apelin, possibly the 3,264 Da protein peak observed, was significantly increased in the EGFR-TKIs resistant group compared with the EGFR-TKIs sensitive group. Therefore, it is possible that high apelin expression may be associated with EGFR-TKIs resistance by affecting angiogenesis.

TYROBP, also known as DNAX-activation protein 12 , is primarily expressed in macrophages, natural killer cells and myeloid cells (32). TYROBP has a preventive function in various diseases due to its ability to modulate immune cells via the activation and inhibition of immune signals $(33,34)$. TYROBP has also been reported to affect cancer metastasis (35). In a previous study, aberrant expression of TYROBP was observed in lung cancer (36). Considering the high expression of TYROBP in the EGFR-TKIs sensitive group, it is possible that TYROBP may contribute to the EGFR-TKIs sensitivity of patients with NSCLC.
Big ET-1 is a biological precursor of ET-1. ET-1 has been determined to have a direct effect on the angiogenesis of perivascular and endothelial cells, and to be able to indirectly upregulate the release of vascular endothelial growth factor (37). ET-1 has also been identified as a novel regulator of tumor angiogenesis and may be a potential anti-angiogenic treatment target (38). Furthermore, the overexpression of plasma big ET-1 and ET-1 levels have been previously detected in NSCLC $(39,40)$. However, in the present study a high expression level of big ET-1 was identified in the EGFR-TKIs sensitive group. Kappers et al (41) previously reported that administration of the EGFR-TKI sunitinib could significantly enhance the serum concentration of ET-1 (41). The ET axis (ETA) and the ET receptor are two distinct receptor subtypes of ET-1, which regulate the downstream effects. Dysregulation of ETA may promote tumour development and progression; therefore, ETA-targeting treatment has been considered as a novel approach for future cancer therapy (42). High expression levels of big ET-1 were identified in the sensitive group; therefore, big ET-1 may regulate ETA activation following EGFR-TKIs treatment of patients with NSCLC.

In conclusion, combining the expression levels of these three proteins may be an effective diagnostic method for EGFR-TKIs resistance. To the best of our knowledge, this study is the first to investigate the diagnostic performance of EGFR-TKIs-associated proteins in serum by combining MB-WCX and MALDI-TOF MS. The predictive model proposed in the present study may provide an improved understanding of the pathogenesis of NSCLC and aid in identifying patient-specific treatments using TKIs. However, despite the high sensitivity and specificity of this predictive model, the number of specimens analyzed in the present study was small, which may limit the validity of the results. Further studies with a larger number of samples are required to verify the current findings. Related experiments should also be performed to confirm the prediction function of the three protein model.

\section{Acknowledgements}

The present study was supported by the Hangzhou Health Science and Technology Plan (grant no. 2012A007) and 

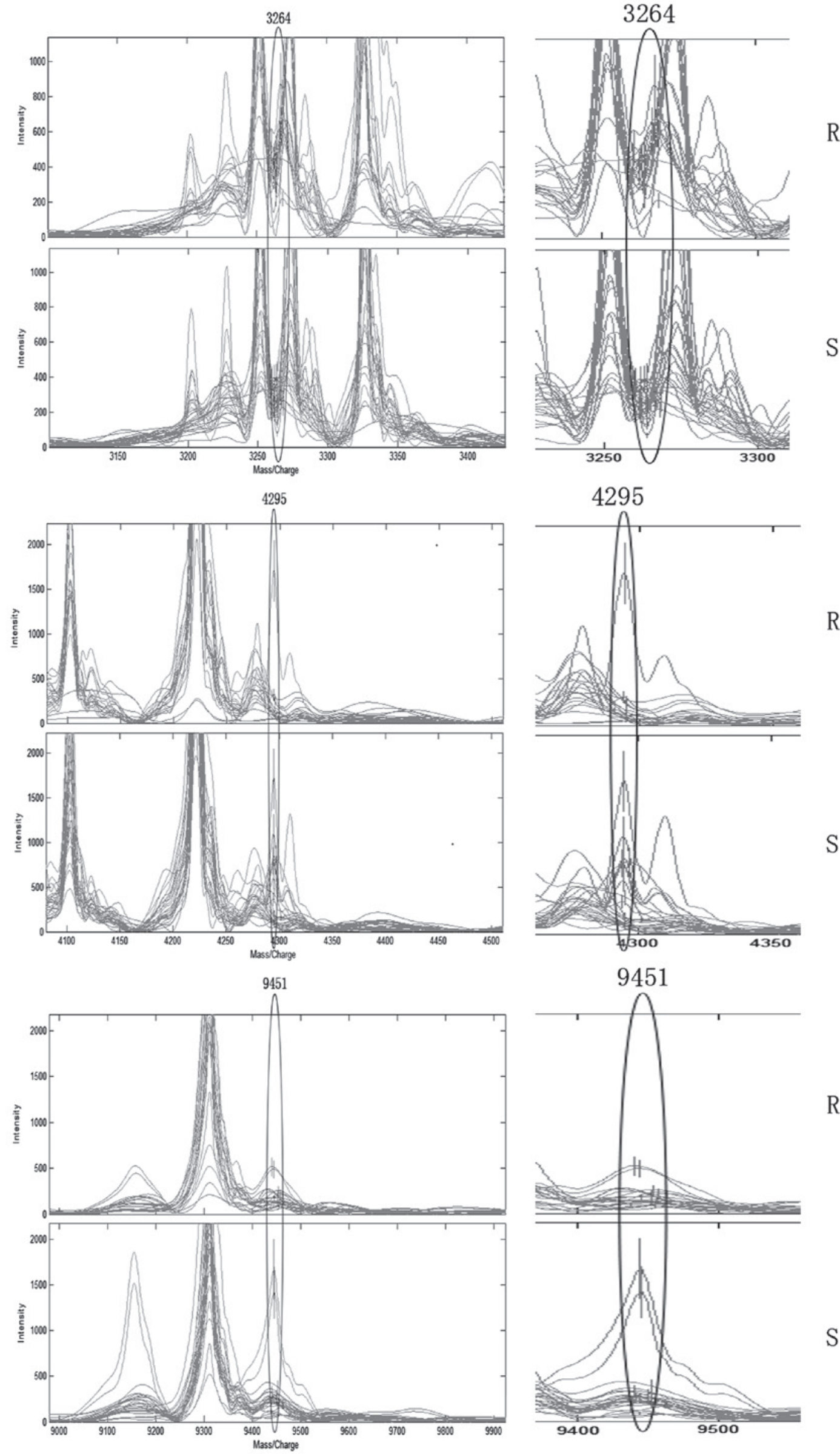

Figure 2. Characteristic peaks observed in patients with NSCLC treated with epidermal growth factor receptor tyrosine kinase inhibitors in S and R groups. The $\mathrm{X}$-axis represents the molecular mass calculation $(\mathrm{m} / \mathrm{z})$, and the $\mathrm{y}$-axis represents relative intensity. NSCLC, non-small cell lung cancer; $\mathrm{S}$, sensitive; R, resistant. 
the Zhejiang Provincial Natural Science Foundation (grant no. LY14H160006).

\section{References}

1. Siegel R, Naishadham D and Jemal A: Cancer statistics, 2012. CA Cancer J Clin 62: 10-29, 2012.

2. Mendelsohn J and Baselga J: Status of epidermal growth factor receptor antagonists in the biology and treatment of cancer. J Clin Oncol 21: 2787-2799, 2003.

3. Fukuoka M, Yano S, Giaccone G, Tamura T, Nakagawa K, Douillard JY, Nishiwaki Y, Vansteenkiste J, Kudoh S, Rischin D, et al: Multi-institutional randomized phase II trial of gefitinib for previously treated patients with advanced non-small-cell lung cancer (The IDEAL 1 Trial) [corrected]. J Clin Oncol 21: 2237-2246, 2003.

4. Lee DH, Han JY, Lee HG, Lee JJ, Lee EK, Kim HY, Kim HK, Hong EK and Lee JS: Gefitinib as a first-line therapy of advanced or metastatic adenocarcinoma of the lung in never-smokers. Clin Cancer Res 11: 3032-3037, 2005.

5. Paez JG, Janne PA, Lee JC, Tracy S, Greulich H, Gabriel S, Herman P, Kaye FJ, Lindeman N, Boggon TJ, et al: EGFR mutations in lung cancer: Correlation with clinical response to gefitinib therapy. Science 304: 1497-1500, 2004.

6. Jackman D, Pao W, Riely GJ, Engelman JA, Kris MG, Jänne PA, Lynch T, Johnson BE and Miller VA: Clinical definition of acquired resistance to epidermal growth factor receptor tyrosine kinase inhibitors in non-small-cell lung cancer. J Clin Oncol 28: 357-360, 2010.

7. Hashida S, Yamamoto H, Shien K, Ohtsuka T, Suzawa K, Maki Y, Furukawa M, Soh J, Asano H, Tsukuda K, et al: Hsp90 inhibitor NVP-AUY922 enhances the radiation sensitivity of lung cancer cell lines with acquired resistance to EGFR-tyrosine kinase inhibitors. Oncol Rep 33: 1499-1504, 2015.

8. Lin Y, Wang $X$ and Jin H: EGFR-TKI resistance in NSCLC patients: Mechanisms and strategies. Am J Cancer Res 4: 411-435, 2014

9. Chen MC, Chen $\mathrm{CH}$, Wang JC, Tsai AC, Liou JP, Pan SL and Teng CM: The HDAC inhibitor, MPT0E028, enhances erlotinib-induced cell death in EGFR-TKI-resistant NSCLC cells. Cell Death Dis 4: e810, 2013.

10. Milan E, Lazzari C, Anand S, Floriani I, Torri V, Sorlini C, Gregorc V and Bachi A: SAA1 is over-expressed in plasma of non small cell lung cancer patients with poor outcome after treatment with epidermal growth factor receptor tyrosine-kinase inhibitors. J Proteomics 76: 91-101, 2012.

11. Yang JJ, Chen HJ, Yan HH, Zhang XC, Zhou Q, Su J, Wang Z, Xu CR, Huang YS, Wang BC, et al: Clinical modes of EGFR tyrosine kinase inhibitor failure and subsequent management in advanced non-small cell lung cancer. Lung Cancer 79: 33-39, 2013.

12. Arcila ME, Oxnard GR, Nafa K, Riely GJ, Solomon SB, Zakowski MF, Kris MG, Pao W, Miller VA and Ladanyi M: Rebiopsy of lung cancer patients with acquired resistance to EGFR inhibitors and enhanced detection of the T790M mutation using a locked nucleic acid-based assay. Clin Cancer Res 17: 1169-1180, 2011

13. Fukui T, Ohe Y, Tsuta K, Furuta K, Sakamoto H, Takano T, Nokihara H, Yamamoto N, Sekine I, Kunitoh $\mathrm{H}$, et al: Prospective study of the accuracy of EGFR mutational analysis by high-resolution melting analysis in small samples obtained from patients with non-small cell lung cancer. Clin Cancer Res 14: 4751-4757, 2008.

14. Weber B, Meldgaard P, Hager H, Wu L, Wei W, Tsai J, Khalil A, Nexo E and Sorensen BS: Detection of EGFR mutations in plasma and biopsies from non-small cell lung cancer patients by allele-specific PCR assays. BMC Cancer 14: 294, 2014.

15. Liu Q, Shi J, Cheng M, Li G, Cao D and Jiang G: Preparation of graphene-encapsulated magnetic microspheres for protein/peptide enrichment and MALDI-TOF MS analysis. Chem Commun (Camb) 48: 1874-1876, 2012.

16. Mantini D, Petrucci F, Pieragostino D, Del Boccio P, Sacchetta P, Candiano G, Ghiggeri GM, Lugaresi A, Federici G, Di Ilio C and Urbani A: A computational platform for MALDI-TOF mass spectrometry data: Application to serum and plasma samples. J Proteomics 73: 562-570, 2010.
17. Yanagisawa K, Shyr Y, Xu BJ, Massion PP, Larsen PH, White BC, Roberts JR, Edgerton M, Gonzalez A, Nadaf S, et al: Proteomic patterns of tumour subsets in non-small-cell lung cancer. Lancet 362: 433-439, 2003.

18. Song QB, Hu WG, Wang P, Yao Y and Zeng HZ: Identification of serum biomarkers for lung cancer using magnetic bead-based SELDI-TOF-MS. Acta Pharmacol Sin 32: 1537-1542, 2011.

19. Liu LH, Shan BE, Tian ZQ, Sang MX, Ai J, Zhang ZF, Meng J, Zhu $\mathrm{H}$ and Wang SJ: Potential biomarkers for esophageal carcinoma detected by matrix-assisted laser desorption/ionization time-of-flight mass spectrometry. Clin Chem Lab Med 48: $855-861,2010$.

20. de Noo ME, Deelder A, van der Werff M, Ozalp A, Mertens B and Tollenaar R: MALDI-TOF serum protein profiling for the detection of breast cancer. Onkologie 29: 501-506, 2006.

21. Mountain CF: Revisions in the international system for staging lung cancer. Chest 111: 1710-1717, 1997.

22. Oken MM, Creech RH, Tormey DC, Horton J, Davis TE, McFadden ET and Carbone PP: Toxicity and response criteria of the eastern cooperative oncology group. Am J Clin Oncol 5: 649-655, 1982.

23. Artimo P, Jonnalagedda M, Arnold K, Baratin D, Csardi G, de Castro E, Duvaud S, Flegel V, Fortier A, Gasteiger E, et al: ExPASy: SIB bioinformatics resource portal. Nucleic Acids Res 40 (Web Server issue): W597-W603, 2012.

24. Fung ET, Wright GL Jr and Dalmasso EA: Proteomic strategies for biomarker identification: Progress and challenges. Curr Opin Mol Ther 2: 643-650, 2000.

25. Skytt A, Thysell E, Stattin P, Stenman UH, Antti H and Wikström P: SELDI-TOF MS versus prostate specific antigen analysis of prospective plasma samples in a nested case-control study of prostate cancer. Int J Cancer 121: 615-620, 2007.

26. Castan-Laurell I, Dray C, Attané C, Duparc T, Knauf C and Valet P: Apelin, diabetes, and obesity. Endocrine 40: 1-9, 2011.

27. Lee DK, Cheng R, Nguyen T, Fan T, Kariyawasam AP, Liu Y, Osmond DH, George SR and O'Dowd BF: Characterization of apelin, the ligand for the APJ receptor. J Neurochem 74: 34-41, 2000.

28. Krist J, Wieder K, Klöting N, Oberbach A, Kralisch S, Wiesner T, Schön MR, Gärtner D, Dietrich A, Shang E, et al: Effects of weight loss and exercise on apelin serum concentrations and adipose tissue expression in human obesity. Obes Facts 6: 57-69, 2013.

29. Sorli SC, Le Gonidec S, Knibiehler B and Audigier Y: Apelin is a potent activator of tumour neoangiogenesis. Oncogene 26: 7692-7699, 2007.

30. Berta J, Kenessey I, Dobos J, Tovari J, Klepetko W, Jan Ankersmit H, Hegedus B, Renyi-Vamos F, Varga J, Lorincz Z, et al: Apelin expression in human non-small cell lung cancer: Role in angiogenesis and prognosis. J Thorac Oncol 5: 1120-1129, 2010.

31. Antushevich H, Pawlina B, Kapica M, Krawczynska A, Herman AP, Kuwahara A, Kato I and Zabielski R: Influence of fundectomy and intraperitoneal or intragastric administration of apelin on apoptosis, mitosis, and DNA repair enzyme OGG1,2 expression in adult rats gastrointestinal tract and pancreas. J Physiol Pharmacol 64: 423-428, 2013.

32. Bouchon A, Hernandez-Munain C, Cella M and Colonna M: A DAP12-mediated pathway regulates expression of CC chemokine receptor 7 and maturation of human dendritic cells. J Exp Med 194: 1111-1122, 2001.

33. Takaki R, Watson SR and Lanier LL: DAP12: An adapter protein with dual functionality. Immunol Rev 214: 118-129, 2006

34. Ma J, Jiang T, Tan L and Yu JT: TYROBP in Alzheimer's disease. Mol Neurobiol 51: 820-826, 2015.

35. Sfakianakis S, Bei ES, Zervakis M, Vassou D and Kafetzopoulos D: On the identification of circulating tumor cells in breast cancer. IEEE J Biomed Health Inform 18: 773-782, 2014.

36. Zhang L, Ma D, Li X, Deng C, Shi Q, You X, Leng X, Li M, Tang F, Zhang F and Li Y: Gene expression profiles of peripheral blood mononuclear cells in primary biliary cirrhosis. Clin Exp Med 14: 409-416, 2014.

37. Spinella F, Caprara V, Di Castro V, Rosanò L, Cianfrocca R, Natali PG and Bagnato A: Endothelin-1 induces the transactivation of vascular endothelial growth factor receptor-3 and modulates cell migration and vasculogenic mimicry in melanoma cells. J Mol Med (Berl) 91: 395-405, 2013.

38. Rosanò L, Spinella F and Bagnato A: Endothelin 1 in cancer: Biological implications and therapeutic opportunities. Nat Rev Cancer 13: 637-651, 2013. 
39. Zuco V, Cassinelli G, Cossa G, Gatti L, Favini E, Tortoreto M, Cominetti D, Scanziani E, Castiglioni V, Cincinelli R, et al: Targeting the invasive phenotype of cisplatin-resistant non-small cell lung cancer cells by a novel histone deacetylase inhibitor. Biochem Pharmacol 94: 79-90, 2015.

40. Arun C, DeCatris M, Hemingway DM, London NJ and O'Byrne KJ: Endothelin-1 is a novel prognostic factor in non-small cell lung cancer. Int J Biol Markers 19: 262-267, 2004
41. Kappers MH, Smedts FM, Horn T, van Esch JH, Sleijfer S, Leijten F, Wesseling S, Strevens H, Jan Danser AH and van den Meiracker AH: The vascular endothelial growth factor receptor inhibitor sunitinib causes a preeclampsia-like syndrome with activation of the endothelin system. Hypertension 58: 295-302, 2011.

42. Bagnato A, Loizidou M, Pflug BR, Curwen J and Growcott J: Role of the endothelin axis and its antagonists in the treatment of cancer. Br J Pharmacol 163: 220-233, 2011. 